\title{
Vasculitis associated with the use of an intrauterine device: A case report
}

\author{
AURELIAN MIHAI GHITA ${ }^{1,2^{*}}$, CRISTINA ALEXANDRESCU ${ }^{3}$, SANZIANA ISTRATE $^{3 *}$, \\ ANCA EVSEI $^{4}$ and ANA CRISTINA GHITA ${ }^{2 *}$ \\ ${ }^{1}$ Department of Physiology II, Faculty of Medicine, 'Carol Davila' University of Medicine and Pharmacy, 050474 Bucharest; \\ ${ }^{2}$ Department of Ophthalmology, Ocularcare Eye Clinic, 011204 Bucharest; ${ }^{3}$ Department of Ophthalmology, \\ Faculty of Dentistry, 'Carol Davila' University of Medicine and Pharmacy, 050474 Bucharest; \\ ${ }^{4}$ Department of Pathology, 'Sf. Maria’ Clinical Hospital, 011172 Bucharest, Romania
}

Received April 20, 2021; Accepted May 20, 2021

DOI: $10.3892 /$ etm.2021.10712

\begin{abstract}
Retinal vasculitis is a complication associated with a local condition or it can be a retinal expression of a systemic inflammatory disorder, which initially may go unnoticed. Drug-associated vasculitis is frequently difficult to identify, because many patients follow treatments with more than one drug and the route of administration varies. A 35-year-old female patient presented with sudden hearing loss, headache and blurred vision that had started two weeks earlier and had become progressively worse. Ophthalmological examination revealed anterior uveitis, bilateral optic disc swelling and retinal vasculitis. The orbito-cerebral MRI, the CT scan and the serological tests were within normal limits. Unable to identify the cause of the retinal vasculitis, the patient's medical history was reviewed. The patient had recently had a gynecological procedure, where a $13.5 \mathrm{mg}$ levonorgestrel intrauterine contraceptive device was implanted. After the device was removed and methylprednisolone treatment started, the patient presented a visible remission of the symptoms and signs. To the best of our knowledge, there is no case of retinal disease and optic disc edema associated with auditory problems caused by an intrauterine device. A proper examination, correlated with a very thorough medical history, could identify rare diseases and associations, in order to provide adequate medical care.
\end{abstract}

Correspondence to: Dr Aurelian Mihai Ghita, Department of Physiology II, Faculty of Medicine, 'Carol Davila' University of Medicine and Pharmacy, 8 Eroii Sanitari Bvd., 050474 Bucharest, Romania

E-mail: mihai.ghita@ocularcare.ro

*Contributed equally

Key words: vasculitis, hearing loss, immune response, hormonal intrauterine device, bilateral optic disc edema, methylprednisolone pulse therapy

\section{Introduction}

Vasculitis is the inflammation of the blood vessel wall secondary to an abnormal immune response $(1,2)$. Systemic vasculitis determines sufferance and fibrinoid necrosis of the endothelial cells, events followed by vascular leakage and blood clot formation with secondary occlusion $(1,3)$. Eventually, all these vascular events determine retinal ischemia and dysfunction of the organ (3).

Retinal vasculitis can be a complication of a local condition or it can be a retinal expression of a systemic inflammatory disorder, which may initially be unnoticed (3-5). The causes of retinal vasculitis are multiple and often overlap, making both the diagnosis and the treatment options challenging (5). Retinal vasculitis is frequently associated with inflammation of the adjacent tissues, such as the choroid or the vitreous, but sometimes, remote ocular structures also appear to be caught in the inflammatory process (6-8).

Retinal vasculitis can be associated with infectious and non-infectious conditions (3-5). The non-infectious causes of vasculitis include ocular disorders and can be drug-induced, i.e., vasculitis associated with a systemic inflammatory disease and vasculitis associated with malignancies (9-11). Drug-associated vasculitis is frequently difficult to identify, because many patients follow treatments with more than one drug and the route of administration varies (12-15). In addition, patients may forget, neglect or hide drug intake. For instance, an intrauterine device (IUD) is considered a medical device. In terms of prevention of an undesired pregnancy, IUDs are usually considered safe with rare side effects, some of which can be severe (16). Currently, there are two types of IUDs available: The copper IUD, which releases copper ions, and the hormonal IUD, which releases a synthetic form of the progesterone hormone, named levonorgestrel (17). The most frequently cited side effects of hormonal IUDs comprise gynecological disorders, headaches, blood-clotting issues, developing acne and breast tenderness, which lead to a higher rate of treatment discontinuation, more than $24 \%$ after 1 year and $33 \%$ after 2 years $(5,17-19)$. Currently, no previous reports of ocular vasculitis associated with the use of an intrauterine device are available in the literature. 

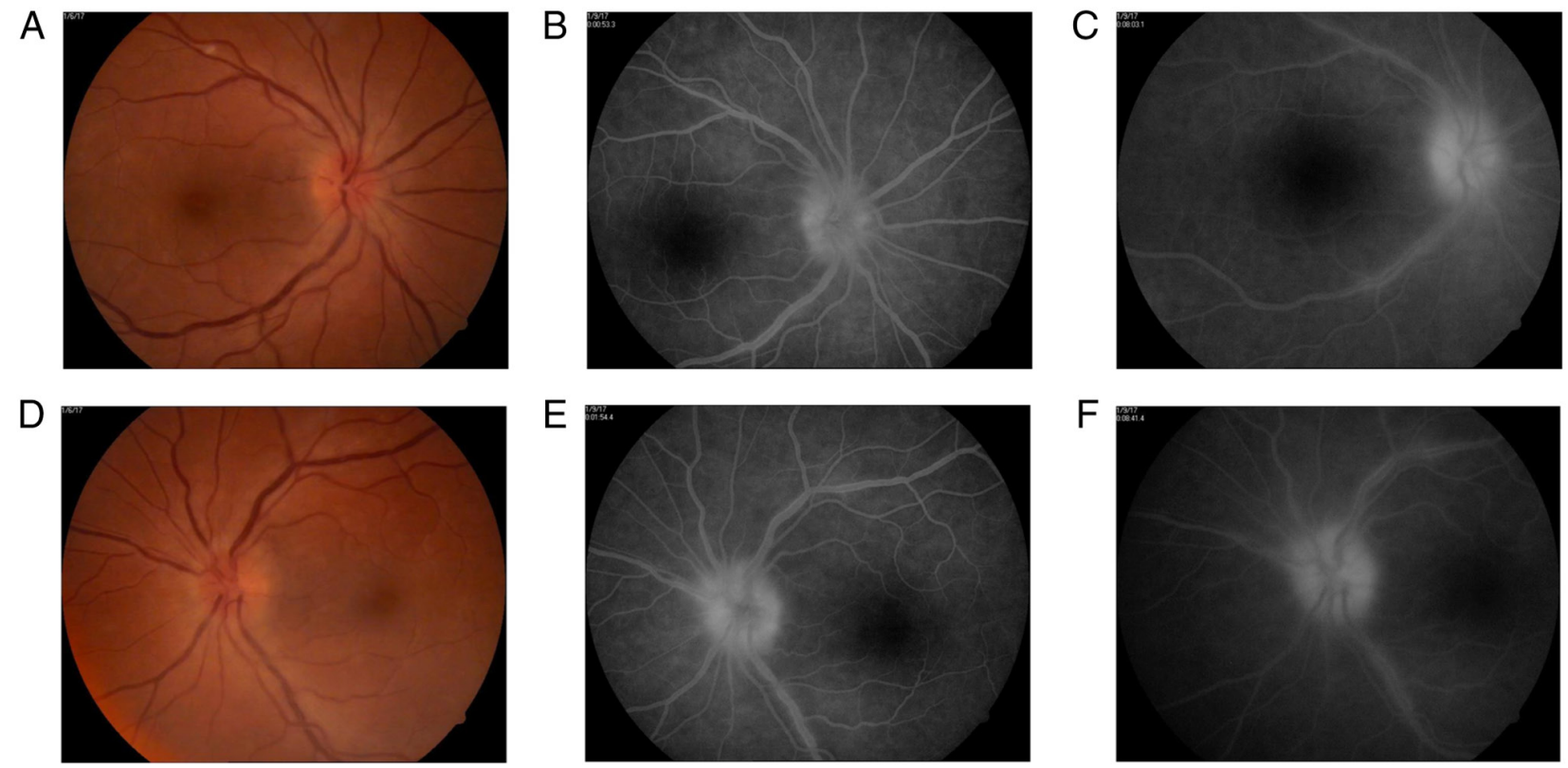

Figure 1. Fundus photography and fluorescein angiography during clinical assessment. (A) The appearance of the posterior pole and (B) fundus angiography in the right eye at $53.3 \mathrm{sec}$. (C) Fundus angiography in the right eye at $8 \mathrm{~min}$ and $3 \mathrm{sec}$. (D) The appearance of the posterior pole in the left eye on fundus photography. (E) Fundus angiography in the left eye at $1 \mathrm{~min}$ and $54 \mathrm{sec}$ and (F) at $8 \mathrm{~min}$ and $41 \mathrm{sec}$.

\section{Case report}

A 35-year-old female patient sought emergency care, complaining of sudden hearing loss, headaches and blurred vision that had started two weeks previously and had gotten progressively worse. The headaches were continuous and located in the occipital area, with episodes of increased pain, accompanied by a decrease in hearing and sometimes dizziness. During the previous two weeks before presentation, the neurological exams had not identified any neurological signs. However, non-steroidal anti-inflammatory drugs were prescribed, but without improvement. Furthermore, the patient was examined by an ear, nose and throat (E.N.T.) doctor, but there were no clinical signs to explain the hearing loss.

Based on the patient's medical history, it was identified that she had a multinodular non-toxic thyroid goiter, no drug allergies, she was a non-smoker and had two natural child births. From the Emergency Room of the University Emergency Hospital Bucharest, the patient was admitted into the neurology department, but still without any detectable clinical neurological signs. The dilated fundus examination revealed bilateral optic disc swelling. An orbito-cerebral magnetic resonance imaging (MRI) and a computed tomography (CT) scan were performed, but with no significant findings. In addition, the blood pressure was normal. Therefore, the patient was discharged from the neurological department and referred to the ophthalmologist.

The ophthalmological assessment revealed a slight decrease in the best corrected visual acuity of 20/30 in the right eye and of 20/25 in the left eye. Slit-lamp examination showed a 1+ faint grade of flare in the anterior chamber, accompanied by a few small corneal endothelium precipitates. Color vision, ocular motility and pupillary light reflex were within normal limits.

Fundus examination reveals bilateral optic disc swelling, hyperemia, loss of the optic cup, optic disc vessels drowned in edema, retinal edema around the optic disc, normal aspect of arteries, but sinuous and dilated veins. The veins also presented a larger diameter than expected and some venous branches were more dilated than others. Their diameter did not constantly decrease towards the periphery, with some medium-sized venous branches greater than the large-sized venous branches (Fig. 1).

Therefore, a fundus fluorescein angiography was carried out, with a delay of 2-3 sec in the appearance of the laminar venous flow and an unequal filling with dye of some temporal medium-sized venous branches. The late phase shows optic disc hyperfluorescence due to venous and capillary leakage. Analyzing the venous branches, segmental diffuse hyperfluorescence in the early phases and staining in the later phases of the angiogram were evident (Fig. 1). No additional retinal or choroidal fluorescein lesions were identified.

In this context, the patient's diagnosis was bilateral retinal vasculitis, papilledema and anterior uveitis, headache and hypoacusis. In order to identify the cause of the retinal vasculitis, various blood tests were performed based on the medical history, symptomatology and clinical assessment, trying to avoid unnecessary and exhaustive investigations. The purpose was to identify an immune or infectious cause of the retinal vasculitis. All serological tests for syphilis, tuberculosis, HSV-1, HSV-2, HZV, CMV, HIV, and ELISA for toxoplasmosis were negative. In addition, IgM and $\operatorname{IgG}$ anti- $\beta 2$ - glycoprotein antibodies, IgM and IgG anti-cardiolipin antibodies, lupus anticoagulant, homocysteine level, antibody anti-DNA double catenary, C-ANCA, P-ANCA, anti-Ro/SSA and anti-La/SSB antibodies, ANA, and rheumatoid factor were negative (Table I). Full blood count, biochemical blood profile and cerebrospinal fluid analysis were non-contributory.

Not being able to identify the cause of the retinal vasculitis, the patient's medical history was reviewed, this time focusing on rheumatological diseases, drug intake or other medical procedures undergone in the recent period. As a result, it was 
Table I. Specific blood tests performed.

\begin{tabular}{|c|c|c|c|}
\hline Immunology & Value & Normal range & Observation \\
\hline Anti- $\beta 2$ glycoprotein antibodies (IgM) (U/ml) & 3.3 & $<5$ & Negative \\
\hline Anti- $\beta 2$ glycoprotein antibodies (IgG) (U/ml) & 1.3 & $<5$ & Negative \\
\hline Anti-cardiolipin Ab (IgG) (U GPL/ml) & $<2$ & $<20$ & Negative \\
\hline Anti-cardiolipin Ab (IgM) (U MPL/ml) & $<2$ & $<20$ & Negative \\
\hline Lupus anticoagulant (ratio) & 0.98 & $<1.2$ & Negative \\
\hline Homocysteine level $\mu \mathrm{mol} / \mathrm{l}$ & 10.1 & $<10$ & $\begin{array}{l}\text { Risk for cardio-vascular } \\
\text { disease, mild }\end{array}$ \\
\hline Anti-DNA double catenary antibodies (U/ml) & 6.6 & $<25$ & Normal \\
\hline Anti C-ANCA antibodies (U/ml) & 1.1 & $<5$ & Negative \\
\hline Anti P-ANCA antibodies (U/ml) & $<2$ & $<13$ & Negative \\
\hline Anti-Ro/SSA antibodies (U/ml) & 2.6 & $<15$ & Negative \\
\hline Anti-La/SSB antibodies (U/ml) & 3.9 & $<15$ & Negative \\
\hline ANA & $<1: 100$ & Titer $0<1: 100$ & Negative \\
\hline Anti-TPO antibodies (U/ml) & 1.44 & $<5.61$ & Normal \\
\hline Anti-thyroglobulin antibodies (UI/ml) & 1 & $<4.11$ & Normal \\
\hline Calcitonin (pg/l) & 2 & $<5$ & Normal \\
\hline Triiodothyronine (T3) (ng/dl) & 0.98 & $0.58-1.59$ & Normal \\
\hline Free thyroxine (T4) (ng/dl) & 0.89 & $0.70-1.48$ & Normal \\
\hline Parathormone (pg/l) & 37.8 & $11-67$ & Normal \\
\hline Erythrocyte sedimentation rate (ESR) $\mathrm{mm} / \mathrm{h}$ & 13 & $<29$ & Normal \\
\hline Rheumatoid factor & & & Negative \\
\hline \multicolumn{4}{|l|}{ Hematology } \\
\hline White blood cells (WBCs) $\left(10^{3} / \mu 1\right)$ & 7.4 & $3.8-11.8$ & Normal \\
\hline Monocytes (\%) & 12.4 & $4.3-10$ & High \\
\hline Red blood cells (RBCs) $\left(10^{6} / \mu 1\right)$ & 4.37 & $3.63-4.92$ & Normal \\
\hline Platelets $\left(10^{3} / \mu 1\right)$ & 290 & $179-408$ & Normal \\
\hline Factor V Leiden & 3.18 & $2.45-3.32$ & Normal \\
\hline Protein C (\%) & 104.42 & $70-140$ & Normal \\
\hline Protein S (\%) & 86.9 & $54.7-123.7$ & Normal \\
\hline \multicolumn{4}{|l|}{ Coagulation } \\
\hline International normalized ratio (INR) & 0.91 & $0.8-1.2$ & Normal \\
\hline $\begin{array}{l}\text { Activated partial thromboplastin time (APTT) } \\
(\mathrm{sec})\end{array}$ & 24.5 & $22-36$ & Normal \\
\hline Fibrinogen mg/dl & 302 & $239-498$ & Normal \\
\hline \multicolumn{4}{|l|}{ Molecular Biology } \\
\hline$P A I-1$ gene mutation & $\begin{array}{l}6754 \mathrm{G} \text { homozygote } \\
844 \mathrm{~A} \text { - AA genotype }\end{array}$ & & $\begin{array}{l}\text { Positive (high risk of thrombophilia } \\
\text { in association with Leiden } \\
\text { V mutation }\end{array}$ \\
\hline MTHFR-gene mutation & $677 \mathrm{C}$ & & Negative \\
\hline & $1298 \mathrm{~A}$ & & Positive \\
\hline \multicolumn{4}{|l|}{ Biochemistry } \\
\hline Alkaline phosphatase (U/l) & 52 & $40-150$ & Normal \\
\hline Total serum calcium (mg/dl) & 8.76 & 8.4-10. & Normal \\
\hline Serum phosphate (mg/dl) & 3.5 & $2.6-4.5$ & Normal \\
\hline Serum magnesium (mg/dl) & 2. & $1.6-2.8$ & Normal \\
\hline
\end{tabular}

Serology

Anti-HIV-1/HIV-2 antibodies

Negative

Anti-HCV antibodies

Negative

Negative

Negative

Antistreptolysin O (ASLO)

Non-reactive

Venereal disease research laboratory (VDRL) 
Table I. Contined.

\begin{tabular}{lccc}
\hline Immunology & Value & Normal range & Observation \\
\hline Rapid plasma reagin (RPR) & & Non-reactive \\
Hepatitis B surface antigen (HBsAg) & & $<0.499$ & Negative \\
Toxoplasmosis (IgM) Index & 0.1 & $<1.6$ & Non-reactive \\
Toxoplasmosis (IgG) (U/ml) & 0.1 & $<0.85$ & Non-reactive \\
Cytomegalovirus (IgM) (S/CO) & 0.05 & $<6$ & Reactive \\
Cytomegalovirus (IgG) (AU/ml) & 250 & $<20$ & Negative \\
HSV-1 (IgM) (Uml) & 7.8 & $<0.9$ & Negative \\
HSV-1 (IgG) Index & 51.1 & $<20$ & Negative \\
Anti HVS2 (IgM) (Uml) & 8.4 & $<0.9$ & Negative \\
Anti-HVS2 (IgG) Index & $<0.5$ & $20-70$ & Negative \\
Angiotensin convertase enzyme (ACE) (U/l) & 17 & & \\
\hline
\end{tabular}

HSV-1, herpes simplex virus type 1; HSV-2, herpes simplex virus type 2; HZV, herpes zoster virus; CMV, cytomegalovirus; HIV, human immunodeficiency virus; ELISA, enzyme-linked immunosorbent assay for toxoplasmosis; IgM, immunoglobulin M; IgG, immunoglobulin G; aCL, anti-cardiolipin antibodies; C-ANCA, anti-neutrophil cytoplasmic antibodies; P-ANCA perinuclear anti-neutrophil cytoplasmic antibodies; ANA, antinuclear antibodies.
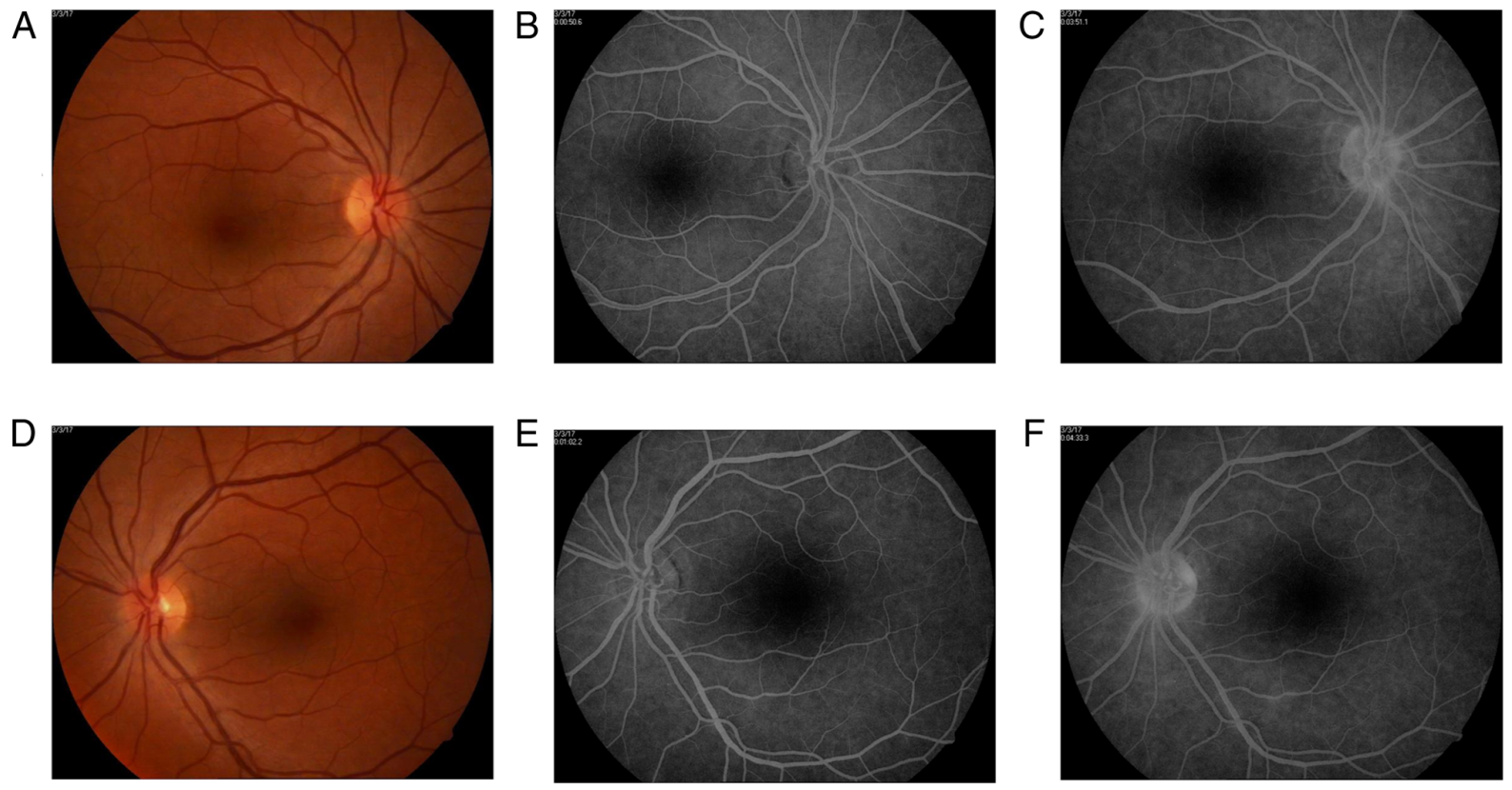

Figure 2. Fundus photography and fluorescein angiography after the IUD was removed and steroid treatment started. (A) The appearance of the posterior pole in the right eye on fundus photography and (B) fundus angiography in the right eye at $50.6 \mathrm{sec}(\mathrm{C})$ and at $3 \mathrm{~min}$ and $51 \mathrm{sec}$. (D) Appearance of the posterior pole in the left eye on fundus photography. (E) Fundus angiography in the left eye at $1 \mathrm{~min}$ and $2 \mathrm{sec}$ and (F) at $4 \mathrm{~min}$ and $33 \mathrm{sec}$.

identified that two weeks prior to onset of the symptoms and one month prior to her presentation at the Emergency Room, the patient underwent a gynecological procedure, in which a $13.5 \mathrm{mg}$ levonorgestrel intrauterine contraceptive device (Bayer Inc.) was implanted.

Systemic vasculitis secondary to the IUD was subsequently considered the final diagnosis. After the IUD was removed, treatment with methylprednisolone pulse therapy (MPPT) of $500 \mathrm{mg} /$ daily for 5 consecutive days commenced. Throughout the treatment, the patient was closely monitored for any possible side effects. After the pulse therapy was completed, the patient was discharged from the University Emergency
Hospital Bucharest, Ophthalmology Clinic and continued the oral treatment with methylprednisolone at $0.8 \mathrm{mg} / \mathrm{kg} / \mathrm{daily}$ with a gradual decrease of the doses every 3 days at home. During the treatment with corticosteroids, the patient also received a proton-pump inhibitor once daily.

One week later, we re-evaluated the patient. All the previous neurological symptoms disappeared and the hearing loss was completely recovered. At the anterior pole examination, there was improvement, the corneal endothelial edema was remitted and the keratic precipitates were reduced in number and size. The fundus examination revealed a decrease of the optic disc edema and in the diameter of the retinal veins 
Table II. The Naranjo adverse drug reaction (ADR) probability scale for the intrauterine contraceptive device used.

Questions

Yes No Do not know Score

1. Are there previous conclusive reports on this reaction?

2. Did the adverse event appear after the suspected drug was readministered?

3. Did the adverse reaction improve when the drug was discontinued or was a specific antagonist administered?

4. Did the adverse reaction reappear when the drug was readministered?

5. Are there alternative causes (other than the drug) that, on their own, could have caused

$\begin{array}{llll}+1 & 0 & 0 & 1 \\ +2 & 0 & 0 & 2 \\ +1 & 0 & 0 & 1\end{array}$

the reaction?

6. Did the reaction reappear when a placebo was given?

$\begin{array}{llll}0 & 0 & 0 & 0 \\ 0 & 2 & 0 & 2\end{array}$

7. Was the blood detected in the blood (or other fluids) in concentrations known to be toxic?

8 . Was the reaction more severe when the dose was increased or less severe when the dose was decreased?

9. Did the patient have a similar reaction to the same or similar drugs in any previous exposure?

10. Was the adverse event confirmed by any objective evidence?

Total

$\begin{array}{llll}0 & 0 & 0 & 0 \\ 0 & 0 & 0 & 0 \\ 0 & 0 & 0 & 0\end{array}$

$\begin{array}{llll}+1 & 0 & 0 & 1\end{array}$

$+1$

A

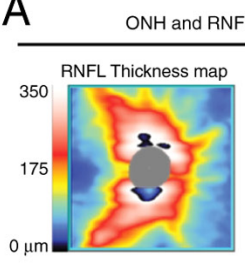

RNFL Deviation map

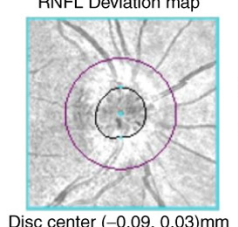

Disc center $(-0.09,0.03) \mathrm{mm}$
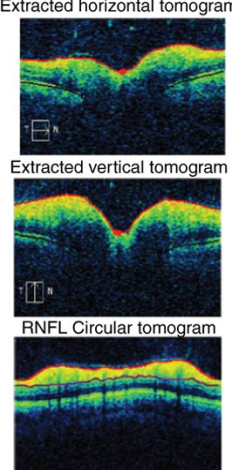

OD $\mathrm{O}$

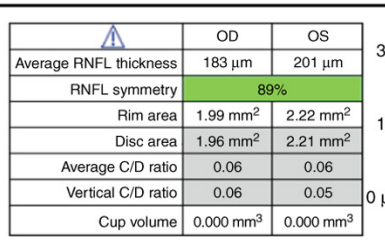

Neuro-retinal rim thickness
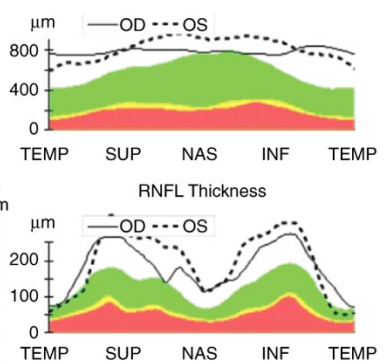

TEMP SUP NAS
Diversified
distribution of norm

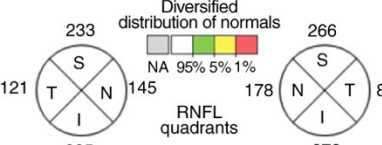

RNFL
quadrants

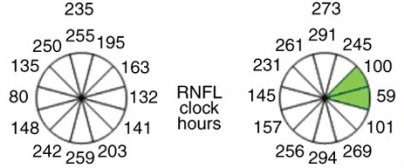

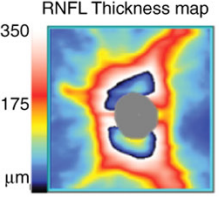

RNFL Deviation map
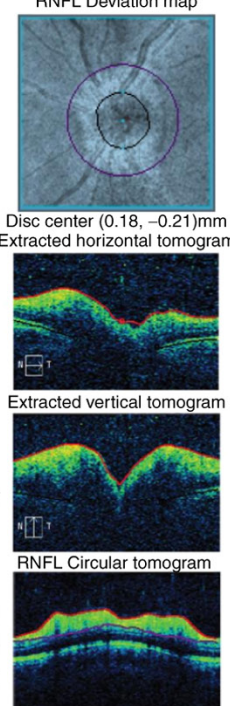

B
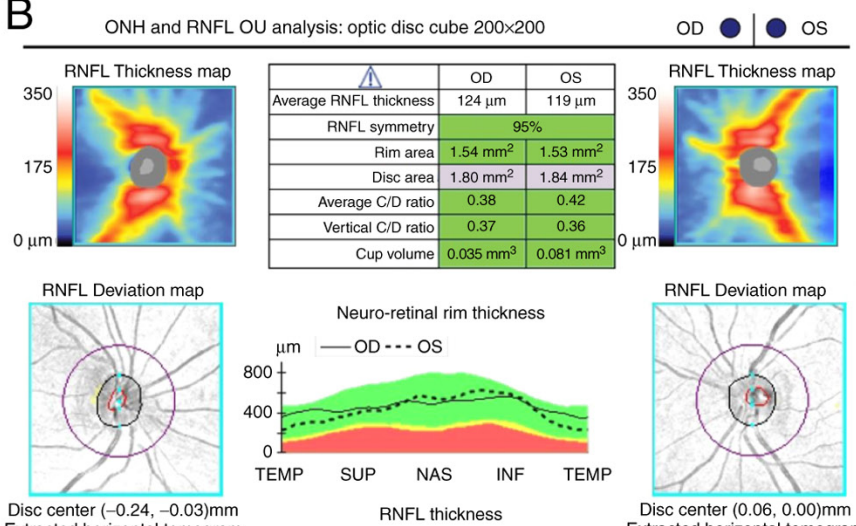

Disc center $(0.06,0.00) \mathrm{mm}$

Disc center $(-0.24,-0.03) \mathrm{mm}$
Extracted horizontal tomogram

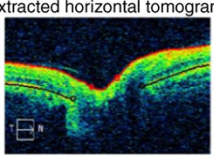

um $-\mathrm{OD} \cdots \mathrm{OS}$

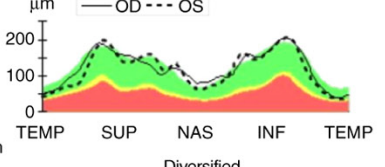

157 distribution of normals 155
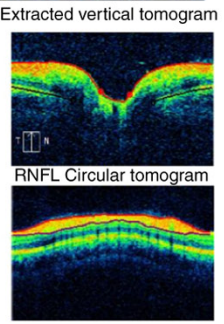

$70 \mathrm{~T} N \mathrm{~N}_{100}$ RNFL
quadrants
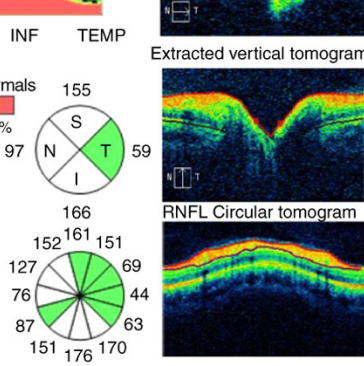

Figure 3. The OCT examination (A) prior to and (B) after one month of treatment.

and their branches (Fig. 2). The optical coherence tomography (OCT) confirmed the identified clinical aspects (Fig. 3). One month later, fundus fluorescein angiography presented with a decrease of the optic disc hyperfluorescence and fewer venous segments of slightly diffuse hyperfluorescence secondary to decreased vascular leakage (Fig. 2).

Ethics approval was obtained from the University Emergency Hospital (Bucharest, Romania). The patient provided written informed consent.

\section{Discussion}

First, the predominant optic disc edema accompanied by very fine ocular signs suggested a neurological condition, misleading the clinicians. However, a careful examination related to ancillary tests including fundus fluorescein angiography and OCT provides the information necessary for a proper diagnosis $(4,20)$. Rarely, retinal vasculitis may be isolated, and idiopathic without any other signs. Typical, retinal vasculitis 
is a manifestation of a systemic disease or of a retinal inflammatory condition. We followed up the guides in order to determine the cause of the disease; however, the tests did not offer the information required. A very thorough examination of the medical history supplied the missing piece of information that was necessary to elucidate the cause. To the best of our knowledge, medical literature has not previously reported any case of retinal vasculitis related to Jaydess ${ }^{\circledR} 13.5 \mathrm{mg}$ levonorgestrel intrauterine contraceptive device (21). However, some studies in literature indicate the occurrence of vasculitis related to oral contraceptive administration, but with mild vascular involvement $(16,18,22,23)$. Mosovich et al published a case of necrotizing vasculitis caused by the Microgynon pills (levonorgestrel, $0.15 \mathrm{mg}$ and ethinyl-oestradiol, $0.03 \mathrm{mg}$ ) (13).

The diagnosis of generalized vasculitis with retinal involvement is, similar to other drug-induced vasculitis, based on the temporal relationship between the drug administration and the appearance of the clinical signs of disease (24). In addition, the absence of other causes that could explain the clinical picture, as systemic autoimmune diseases or infectious diseases (the ancillary test was negative or non-reactive), the remission of the disease after the device has been removed and the positive response to the systemic steroid therapy also indicate a direct relationship between the IUD and disease.

In addition, we focused on thyroid disorders in order to exclude a secondary cause, being aware of the possible relationship with primary or secondary ANCA vasculitis. The test results excluded other retinal vasculitis associated with systemic inflammatory diseases, such as systemic lupus erythematosus, Wegener granulomatosis, microscopic polyangiitis, antiphospholipid syndrome and ANCA-associated vasculitis $(25,26)$.

As the patient was complaining of hearing dysfunction, and in the context of a mild anterior uveitis, Cogan syndrome was suspected (27). However, in the absence of any cardiac signs, normal blood level of leukocytes, a slight increase of the erythrocyte sedimentation rate, a normal C-reactive protein, we excluded this syndrome $(27,28)$.

Finally, the Naranjo adverse drug reaction (ADR) probability scale was performed and suggested an outcome to our clinical evidence $(29,30)$ (Table II). The last question is slightly contentious, since it was based on the patient's response. The patient claimed that when she was previously under treatment with similar drugs, such as oral contraceptive (similar to levonorgestrel), she presented a low intensity similar response, but could not provide medical evidence to support these aspects.

The Naranjo criteria classifies the probability that an adverse event is related to a specific drug therapy, based on a list of weighted questions, which examine factors such as the temporal association of drug administration and the event, alternative causes that can explain the event, drug levels, dose-response relationship and the patient's previous experience with that drug (29-31). If ADR score is $\geq 9$, the adverse drug reaction is considered as definite, if the score is between 5 and 8 it is interpreted as probable, possible for a score between 1 and 4, and doubtful if the score is 0 (31). The Naranjo criteria does not take into account drug interactions. Drugs are evaluated individually for causality, and points are deducted if another factor may have resulted in the adverse event, thereby weakening the causal association.
In conclusion, to the best of our knowledge no previous case of retinal disease and optic disc edema associated with auditory problems (possible vascular) caused by an intrauterine device has been reported. A proper examination correlated with a very thorough medical history could identify rare diseases and associations, in order to provide adequate medical care.

\section{Acknowledgements}

Professional editing, linguistic and technical assistance were performed by Irina Radu.

\section{Funding}

No funding was received.

\section{Availability of data and materials}

Further information regarding the case is available from the corresponding author upon reasonable request.

\section{Authors' contributions}

AMG had a substantial contribution to the conception and design of the work, analyzed and interpreted the data being also the first investigator and coordinator of the article. AMG also gave the final approval of the version to be published. CA collected all the data from the patient's medical history and produced the figures and tables. SI performed ocular coherence tomography, fundus fluorescein angiography, and verified that the information and data were accurate. AE performed the Naranjo adverse drug reaction (ADR) probability scale and revised the paper. ACG was responsible for literature research of the current data, drafted the study, and revised it critically for important intellectual content. AMG and ACG assessed the authenticity of all the raw data. All authors read and approved the final manuscript.

\section{Ethics approval and consent to participate}

Ethics approval was obtained from the University Emergency Hospital, 050098 Bucharest, Romania. The patient provided written informed consent.

\section{Patient consent for publication}

Not applicable.

\section{Competing interests}

The authors declare that they have no competing interests.

\section{References}

1. Guillevin L and Dorner T: Vasculitis: Mechanisms involved and clinical manifestations. Arthritis Res Ther 9 (Suppl 2): S9, 2007.

2. Langford CA: Vasculitis. J Allergy Clin Immunol 125 (Suppl 2): S216-S225, 2010

3. Trese MGJ, Yonekawa Y, Thomas BJ and Randhawa S: Vasculitic central retinal vein occlusion: The presenting sign of seronegative rheumatoid arthritis. Am J Ophthalmol Case Rep 2: 26-29, 2016. 
4. Rosenbaum JT, Sibley CH and Lin P: Retinal vasculitis. Curr Opin Rheumatol 28: 228-235, 2016.

5. Jennette JC: Overview of the 2012 revised International chapel hill consensus conference nomenclature of vasculitides. Clin Exp Nephrol 17: 603-606, 2013.

6. Apinyawasisuk S, Rothova A, Kunavisarut P and Pathanapitoon K: Clinical features and etiology of retinal vasculitis in Northern Thailand. Indian J Ophthalmol 61: 739-742, 2013.

7. Fukunaga H, Kaburaki T, Shirahama S, Tanaka R, Murata H, Sato T, Takeuchi M, Tozawa H, Urade Y, Katsura M, et al: Analysis of inflammatory mediators in the vitreous humor of eyes with pan-uveitis according to aetiological classification. Sci Rep 10: 2783, 2020.

8. Kaburaki T, Fukunaga H, Tanaka R, Nakahara H, Kawashima H, Shirahama S, Izawa H, Komae K, Takamoto M, Soga H and Aihara M: Retinal vascular inflammatory and occlusive changes in infectious and non-infectious uveitis. Jpn J Ophthalmol 64 $150-159,2020$.

9. Agarwal A, Karkhur S, Aggarwal K, Invernizzi A, Singh R, Dogra MR, Gupta V, Gupta A, Do DV and Nguyen QD Epidemiology and clinical features of inflammatory retinal vascular occlusions: Pooled data from two tertiary-referral institutions. Clin Exp Ophthalmol 46: 62-74, 2018.

10. Grau RG: Drug-induced vasculitis: New insights and a changing lineup of suspects. Curr Rheumatol Rep 17: 71, 2015.

11. Iwahashi $\mathrm{C}$, Ono $\mathrm{H}$, Haruta $\mathrm{M}$, Minami $\mathrm{T}$, Mashimo $\mathrm{H}$, Shimojo H and Ohguro N: New onset or exacerbation of uveitis with infliximab: Paradoxical effects? BMJ Open Ophthalmol 4: e000250, 2019

12. Agarwal A, Pilania RK, Anjani G, Choudhary H, Gupta A and Gupta V: Retinal vasculitis with coats-like response in a young girl with Parry-Romberg syndrome. J Clin Rheumatol: May 21, 2019 (Epub ahead of print).

13. Mosovich B, Biton A and Avinoach I: Vasculitis with cutaneous necrosis induced by oral contraceptive. Harefuah 120: 451-453, 1991 (In Hebrew)

14. Trusau A and Brit ML: Propylthiouracil-induced ANCA-negative cutaneous small vessel vasculitis. J Community Hosp Intern Med Perspect 8: 35-37, 2018

15. Wiik A: Drug-induced vasculitis. Curr Opin Rheumatol 20: 35-39, 2008.

16. Kailasam C and Cahill D: Review of the safety, efficacy and patient acceptability of the levonorgestrel-releasing intrauterine system. Patient Prefer Adherence 2: 293-302, 2008.

17. Andersson K, Odlind V and Rybo G: Levonorgestrel-releasing and copper-releasing (Nova T) IUDs during five years of use: A randomized comparative trial. Contraception 49: 56-72, 1994.
18. Sturridge F and Guillebaud J: A risk-benefit assessment of the levonorgestrel-releasing intrauterine system. Drug Saf 15: 430-440, 1996

19. Vrettakos C and Bajaj T: Levonorgestrel. In: StatPearls [Internet] Treasure Island (FL): StatPearls Publishing; 2021.

20. Abu El-Asrar AM, Herbort CP and Tabbara KF: Differential diagnosis of retinal vasculitis. Middle East Afr J Ophthalmol 16: 202-218, 2009.

21. Wildemeersch D, Andrade A, Goldstuck ND, Hasskamp T and Jackers G: Intrauterine levonorgestrel delivery with frameless fibrous delivery system: Review of clinical experience. Int J Womens Health 9: 49-58, 2017.

22. Fekete GL and Fekete L: Cutaneous leukocytoclastic vasculitis associated with erlotinib treatment: A case report and review of the literature. Exp Ther Med 17: 1128-1131, 2019.

23. Mansour D: The benefits and risks of using a levonorgestrel-releasing intrauterine system for contraception. Contraception 85: 224-234, 2012

24. Radic M, Martinović Kaliterna D and Radic J: Drug-induced vasculitis: A clinical and pathological review. Neth J Med 70: 12-17, 2012.

25. Papaliodis GN: Ophthalmologic manifestations of systemic vasculitis. Curr Opin Ophthalmol 28: 613-616, 2017.

26. Tugal-Tutkun I: Systemic vasculitis and the eye. Curr Opin Rheumatol 29: 24-32, 2017

27. Iliescu DA, Timaru CM, Batras M, De Simone A and Stefan C: Cogan's syndrome. Rom J Ophthalmol 59: 6-13, 2015.

28. Vollertsen RS: Vasculitis and Cogan's syndrome. Rheum Dis Clin North Am 16: 433-439, 1990.

29. Behera SK, Das S, Xavier AS, Velupula S and Sandhiya S: Comparison of different methods for causality assessment of adverse drug reactions. Int J Clin Pharm 40: 903-910, 2018

30. Murayama H, Sakuma M, Takahashi $\mathrm{Y}$ and Morimoto $\mathrm{T}$ : Improving the assessment of adverse drug reactions using the Naranjo Algorithm in daily practice: The Japan adverse drug events study. Pharmacol Res Perspect 6: e00373, 2018.

31. Naranjo CA, Busto U, Sellers EM, Sandor P, Ruiz I, Roberts EA, Janecek E, Domecq C and Greenblatt DJ: A method for estimating the probability of adverse drug reactions. Clin Pharmacol Ther 30: 239-245, 1981

This work is licensed under a Creative Commons Attribution-NonCommercial-NoDerivatives 4.0 International (CC BY-NC-ND 4.0) License. 